\title{
ROBUST HETEROCLINIC TANGENCIES
}

\author{
PABLO G. BARRIENTOS AND SEBASTIÁN A. PÉREZ
}

\begin{abstract}
Авsтract. We construct diffeomorphisms in dimension $d \geq 2$ exhibiting $C^{1}$-robust heteroclinic tangencies.
\end{abstract}

\section{INTRODUCTION}

An important problem in the modern theory of Dynamical Systems is to describe diffeomorphisms whose qualitative behavior exhibits robustness under (small) perturbations and how abundant these sets of dynamics can be. Motivated by this issue, Smale introduced in [15] the hyperbolic diffeomorphisms as examples of structural stable dynamics (open sets of dynamics which are all of them conjugated). However, the transverse intersection between the invariant manifolds of basic sets was soon observed as a necessary condition $[20,13,17]$. The main goal of this article is to study the persistence of the non-transverse intersection between those manifolds. Namely, we focus in tangencial heteroclinic orbits.

A diffeomorphism $f$ of a manifold $\mathcal{M}$ has a heteroclinic tangency if there are different transitive hyperbolic sets $\Lambda$ and $\Gamma$, points $P \in \Lambda, Q \in \Gamma$ and $Y \in W^{u}(P) \cap W^{s}(Q)$ such that

$$
c_{T} \stackrel{\text { def }}{=} \operatorname{dim} \mathcal{M}-\operatorname{dim}\left[T_{Y} W^{u}(P)+T_{Y} W^{s}(Q)\right]>0 \quad \text { and } \quad d_{T} \stackrel{\text { def }}{=} \operatorname{dim} T_{Y} W^{u}(P) \cap T_{Y} W^{s}(Q)>0 .
$$

The number $c_{T}$ is called codimension of the tangency and measures how far the tangencial intersection is from a transverse intersection. On the other hand, $d_{T}$ indicates the number of linearly independent common tangencial directions. Observe that

$$
c_{T}=d_{T}-k_{T} \quad \text { with } \quad k_{T}=\operatorname{ind}(\Lambda)-\operatorname{ind}(\Gamma)
$$

where ind $(\Sigma)$ denotes the stable index of a (transitive) hyperbolic set $\Sigma$. The integer $k_{T}$ is called signed co-index. Notice that when $k_{T}>0$ this number coincides with the classical co-index between $\Lambda$ and $\Gamma$. Moreover, $k_{T}>0$ if and only if

$$
\operatorname{dim} T_{Y} W^{u}(P)+\operatorname{dim} T_{Y} W^{s}(Q)>\operatorname{dim} M .
$$

If $k_{T}=0$, the heteroclinic tangency is called equidimensional and otherwise heterodimensional. Figure 1 illustrates the different types of heteroclinic tangencies in dimension three.

Heterodimensional tangencies with signed co-index $k_{T}>0$ was introduced in [9] where interesting dynamics consequences were obtained. Indeed, the authors showed that the

2000 Mathematics Subject Classification. Primary 34D30, 37D10, 37D30, 37 G25.

Key words and phrases. folding manifolds, robust equidimensional tangencies, robust heterodimensional tangencies. 


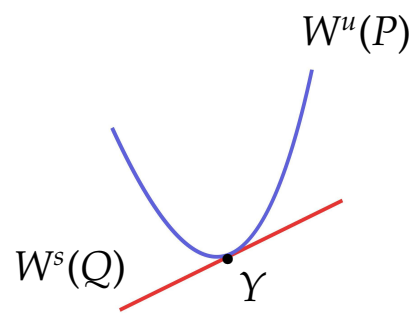

(a) heterodimensional

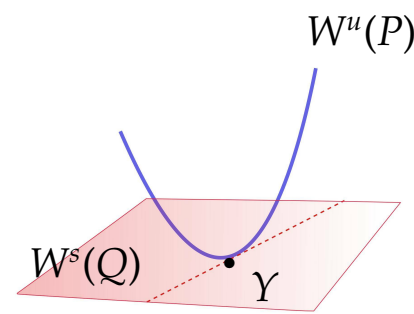

(b) equidimensional

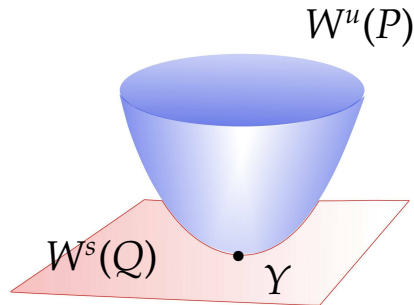

(c) heterodimensional

Figure 1. Heteroclinic tangencies in dimension 3: (a) $c_{T}=2, d_{T}=1$ and $k_{T}=-1 ;(b) c_{T}=1$, $d_{T}=1$ and $k_{T}=0 ;(c) c_{T}=1, d_{T}=2$ and $k_{T}=1$.

$C^{1}$-unfolding of a three dimensional heterodimensional tangency (with $k_{T}=1$ ) leads to $C^{1}$ robustly non-dominated dynamics and in some cases to very intermingled dynamics related to universal dynamics, for details see $[9,6]$. In the $C^{r}$-topologies with $r>1$, the bifurcation of such tangencies leads, for instance, to the existence of blender dynamics [8, 10]. Kiriki and Soma in [12] obtain the first examples of $C^{2}$-robust heterodimensional tangencies with $c_{T}=1$ and $k_{T}=d-2$ in any manifold of dimension $d \geq 3$. Recently in [3] new examples of $C^{2}$-robust heterodimensional tangencies with $0<c_{T} \leq\lfloor(d-3) / 2\rfloor$ and $1 \leq k_{T} \leq d-2-2 c_{T}$ were also constructed in any manifold of dimension $d \geq 5$. In the same work, $C^{2}$-robust heteroclinic tangencies with $k \leq 0$ were also obtained. In [12] it was proposed the problem of constructing $C^{1}$-robust heterodimensional tangencies with $k>0$ in any dimension greater than 2. Motivated by this issue, the main result of this work shows that, in particular, these tangencies can be built persistently under $C^{1}$-perturbations.

Theorem A. Every manifold of dimension $d \geq 2$ admits a diffeomorphism $f$ having a $C^{1}$-robust heteroclinic tangency of codimension $c_{T}=1$ and signed co-index $0 \leq k \leq d-2$.

By constraints of the dimension, in surfaces, we only get equidimensional tangencies. In higher dimensions, we construct both type of heteroclinic tangencies: equidimensional and heterodimensional with all possible signed co-index between 0 and $d-1$.

Theorem A will be proved in Section 2, by providing a local construction close to the classical examples given by Abraham and Smale [1], Simon [14] and Asaoka in [2]. In Section 3 we will give a different proof of Theorem A using ideas of the recent work [4] studying the differential cocyle in the tangent space. These new ideas allow us to generalize the construction for large codimension $\left(c_{T} \geq 2\right)$ in some particular cases. Namely, we get the following result.

Theorem B. Given integers $c_{T} \geq 1$ and $s>c_{T}$, there are diffeomorphisms $f$ of the d-dimensional torus $\mathbb{T}^{d}$ with $d=c_{T} \cdot(s+1)$ having a $C^{1}$-robust heterodimensional tangency of codimension $c_{T}$ and signed co-index $k_{T}=s-c_{T}>0$. 


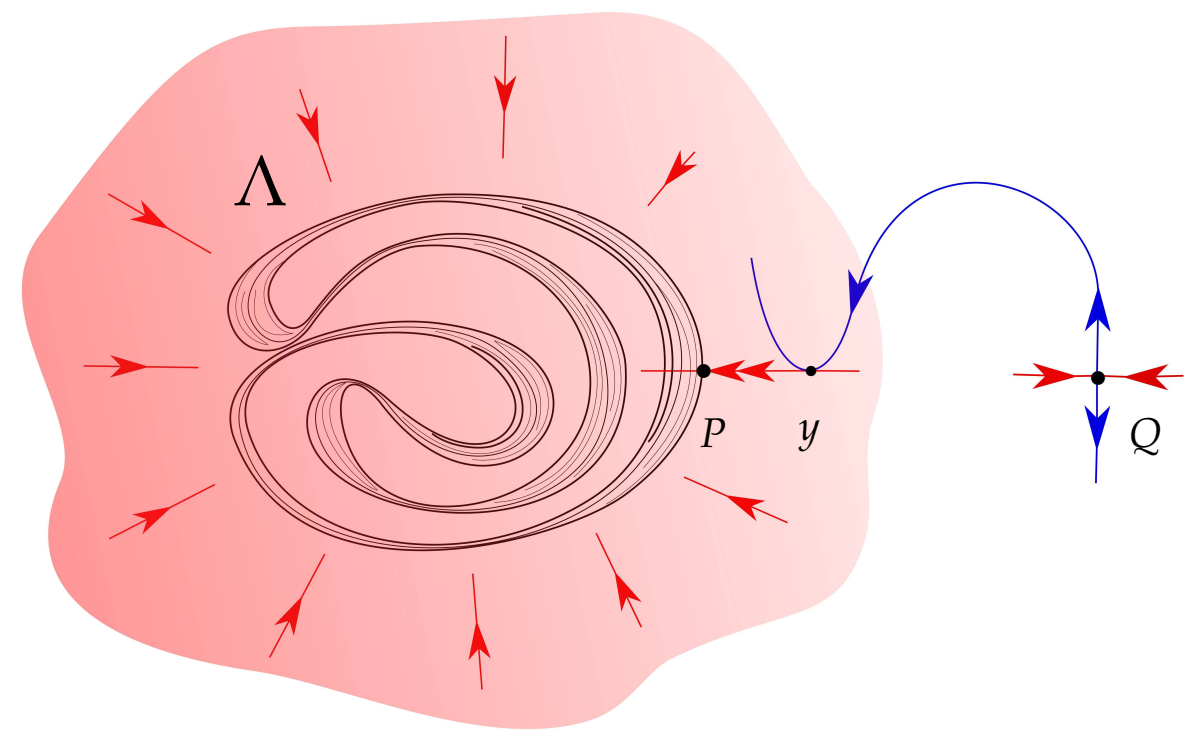

Figure 2. Equidimensional tangency in dimension $d=2$

The proof of the above theorem will be carried on in Section 4. Finally, in Section 5 we conclude the work with a section of open questions and future directions.

\section{Geometric CONSTRUCtion of $C^{1}$-ROBUSt HETEROCLINIC tANGENCIES}

Let $\Lambda$ be a Plykin attractor in a disc with three holes [16]. Let $Q$ be a saddle in the complement of this disc as in Figure 2. To do possible the construction we need to assume that $Q$ belongs to a Plykin repellor $\Gamma$. This figure illustrates the two-dimensional version of the Asaoka's argument [2] (see also [14]) providing a $C^{1}$-robust equidimensional tangency in any surface between the stable manifold of $\Lambda$ and the unstable manifold of $Q$.

Using this idea, we built a diffeomorphism $f$ on any manifold $\mathcal{M}$ of dimension $d \geq 2$ having a hyperbolic attractor $\Lambda$ whose attracting region is a connected set and foliated by $(d-1)$-dimensional stable submanifolds. After that, we consider a fixed point $P$ in $\Lambda$ and another fixed point $Q$ of $f$ of stable index $d-1-k$ where $0 \leq k \leq d-2$ creating a heteroclinic tangency between $W^{s}(P)$ and $W^{u}(Q)$, so that $W^{u}(Q)$ and $W^{s}(\Lambda)$ meet transversely. The $C^{1}-$ persistence of this last intersection provides a $C^{1}$-robust heteroclinic tangency associated with $\Lambda$ and $Q$.

2.1. Construction. We now give the details of our construction. Since our argument is local, we can put $\mathcal{M}=\mathbb{R}^{d}$ with $d \geq 2$. First, we take a two-dimensional diffeomorphism $h$ with a Plykin attractor $\Sigma$ constructed in local coordinates inside a disk $D \subset \mathbb{R}^{2}$ with three holes. We consider a $C^{r}$-diffeomorphism $f: \mathcal{M} \rightarrow \mathcal{M}$ with $r \geq 1$ such that for a small $\varepsilon>0$, the restriction of $f$ to the set $D_{\varepsilon} \stackrel{\text { def }}{=}[-\varepsilon, \varepsilon]^{d-2} \times D$ is given by

$$
f=g \times h \text { where } g(t)=\lambda t \text { for } t \in[-\varepsilon, \varepsilon]^{d-2} \text { and } 0<\lambda<1 \text {. }
$$




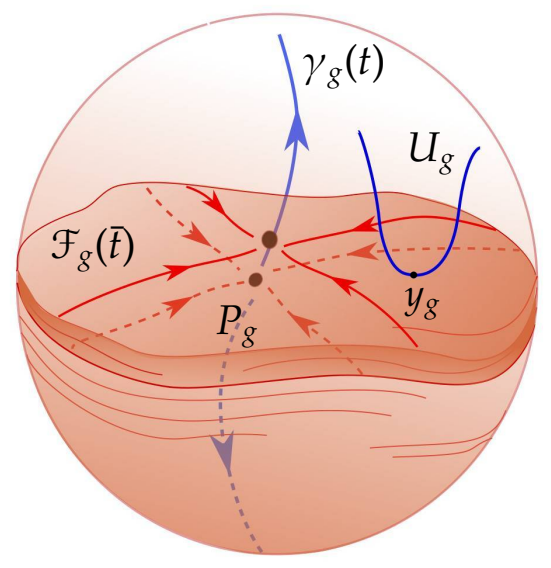

(a)

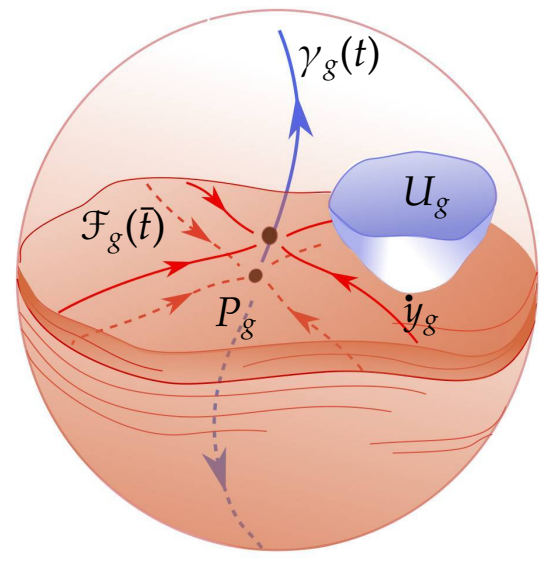

(b)

Figure 3. (a) $C^{1}$-robust equidimensional tangency. (b) $C^{1}$-robust heterodimensional tangency.

Thus, the set

$$
\Lambda \stackrel{\text { def }}{=}\left\{0^{d-2}\right\} \times \Sigma=\bigcap_{n \geqslant 1} f^{n}\left(D_{\varepsilon}\right)
$$

is a hyperbolic attractor of $f$ and $D_{\varepsilon}$ is a trapping region of $f$, i.e. $f\left(D_{\varepsilon}\right) \subset \operatorname{interior}\left(D_{\varepsilon}\right)$. The structural stability of $\Lambda$ provides the existence of a $C^{1}$-neighborhood $\mathcal{V}$ of $f$ such that for each $g \in \mathcal{V}$, the continuation $\Lambda_{g}$ of $\Lambda$ has by trapping region the set $D_{\varepsilon}$. We remark that the local stable manifolds $W_{\text {loc }}^{s}(x)=W^{s}(x) \cap D_{\varepsilon}$ for $x \in \Lambda$ provide a foliation of the set $D_{\varepsilon}$ by leaves (plaques) of dimension $d-1$. It is not hard to verify that this property also holds for any diffeomorphism $g$ in $\mathcal{V}$. We will denote by $W_{l o c}^{s}(x, g)$ the stable local manifold at $x$ for $g$.

Now we build the robust heteroclinc tangency of elliptic type. Recall that a heteroclinic tangency $y \in W^{u}(Q) \cap W^{s}(P)$, is of elliptic type if there is a neighborhood $U$ of $y$ contained in either, $W^{u}(Q)$ or $W^{s}(P)$, say $W^{u}(Q)$, such that any point in $U-\{y\}$ belongs to the same side of the tangent space $T_{y} W^{u}(Q)$. We consider a fixed point $P \in \Lambda$ and a small open ball $B$ centered at $P$ such that $\bar{B}$ is contained in $D_{\varepsilon}$. We observe that for every $g \in \mathcal{V}, B$ is foliated by

$$
\mathcal{F}_{g}(x) \stackrel{\text { def }}{=} W_{l o c}^{s}(x, g) \cap B, \quad x \in \Lambda_{g} .
$$

Consider a hyperbolic fixed point $Q \notin D_{\varepsilon}$ of $f$ with stable index $d-1-k$. By means of a homotopic deformation, we force to the $(k+1)$-dimensional unstable manifold $W^{u}(Q)$ intersects non-transversely the stable manifold $W^{s}(P)$ in a heteroclinic tangency of elliptic type, namely $y$. Taking a suitable iterated if necessary, we can assume that $y$ is in $B$. Thus, this last diffeomorphism, that again we call $f$, has a heteroclinic tangency of codimension $c_{T}=1$ and signed co-index $k_{T}=k$ with $0 \leq k \leq d-2$, associated with the saddles $P \in \Lambda$ and $Q$. 


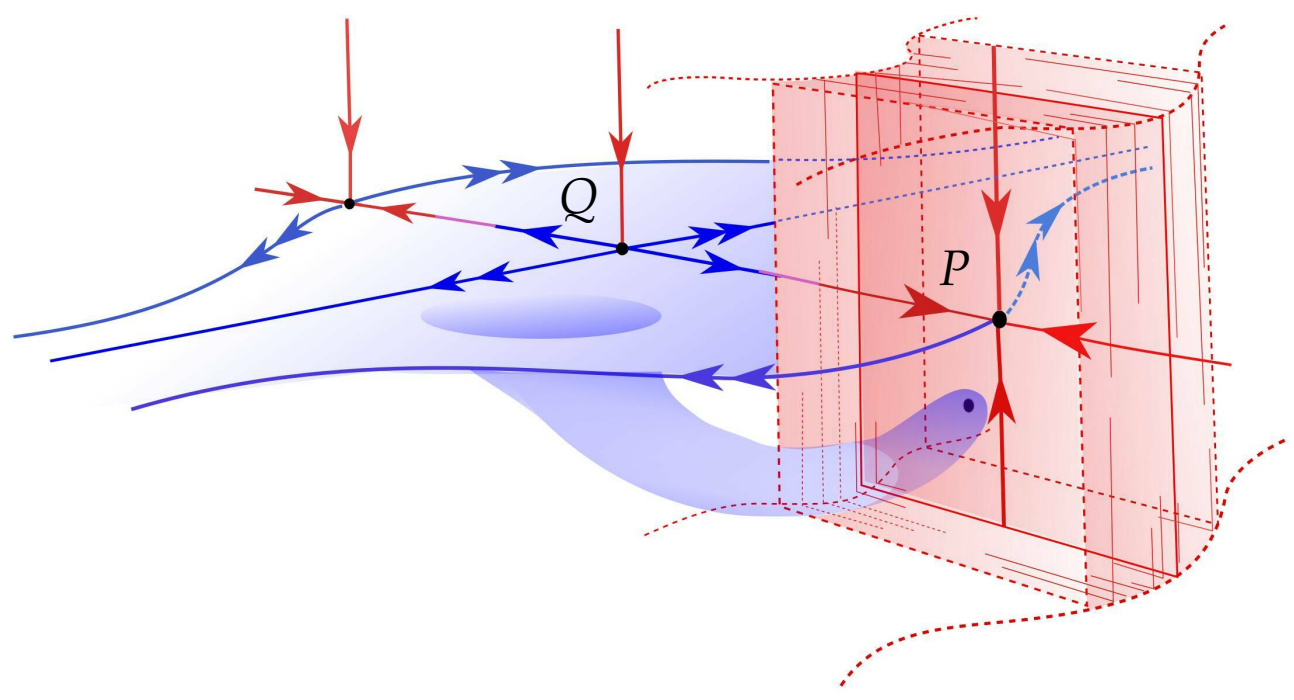

Figure 4. Heterodimensional tangency constructed from a deformation of diffeomorphism $f$ of $\mathbb{T}^{3}$ locally defined as the product $g \times h$ where $h$ has a DA-attractor in $\mathbb{T}^{2}$ and $g$ is a contraction.

On the other hand, by definition, there exists a neighborhood $U$ of $y$ contained in $W^{u}(Q)$ such that $U-\{y\}$ is contained in $W^{s}(\Lambda) \pitchfork W^{u}(Q)$. See Figure 3. We will see that the $C^{1}$ persistence of these last transverse intersections provides a $C^{1}$-robust heteroclinic tangency associated with $\Lambda$ and $Q$. Besides, for each $g \in \mathcal{V}$, we consider a small curve $\gamma_{g}: t \in$ $(-r, r) \mapsto \gamma_{g}(t) \in \Lambda_{g} \subset D_{\varepsilon}$ parameterizing a small local unstable manifold of the continuation $P_{g}=\gamma_{g}(0)$ of $P$ such that

$$
B=\bigcup_{t \in(-r, r)} \mathcal{F}_{g}(t) \quad \text { where } \quad \mathcal{F}_{g}(t)=\mathcal{F}_{g}\left(\gamma_{g}(t)\right)
$$

Since $y$ is a heteroclinic tangency of elliptic type between $W^{u}(Q)$ and $W^{s}(P)$ we can assume that (see Figure 3)

$$
\begin{aligned}
& U \cap \mathcal{F}_{f}(0)=\{y\}, \\
& U \cap \mathcal{F}_{f}(t) \subset W^{u}(Q) \pitchfork W^{s}\left(\gamma_{f}(t)\right) \quad \text { for all } t \in(0, r), \\
& U \cap \mathcal{F}_{f}(t)=\emptyset \text { for all } t \in(-r, 0) .
\end{aligned}
$$

Our conditions imply that for each $g \in \mathcal{V}$, the set

$$
I_{g}=\left\{t \in(-r, r): U_{g} \pitchfork \mathcal{F}_{g}(t) \neq \emptyset\right\}
$$

is inferiorly bounded where $U_{g}$ is a continuation in $W_{l o c}^{s}\left(Q_{g}\right)$ of the neighborhood $U$. Thus, if $\bar{t}$ is the infimum of $I_{g}$ then $U_{g}$ and $\mathcal{F}_{g}(\bar{t})$ meet in a heteroclinic tangency $y_{g}$ of codimension $c_{T}=1$ and signed co-index $k_{T}=k$ with $0 \leq k \leq d-2$. This completes the proof of Theorem A. 

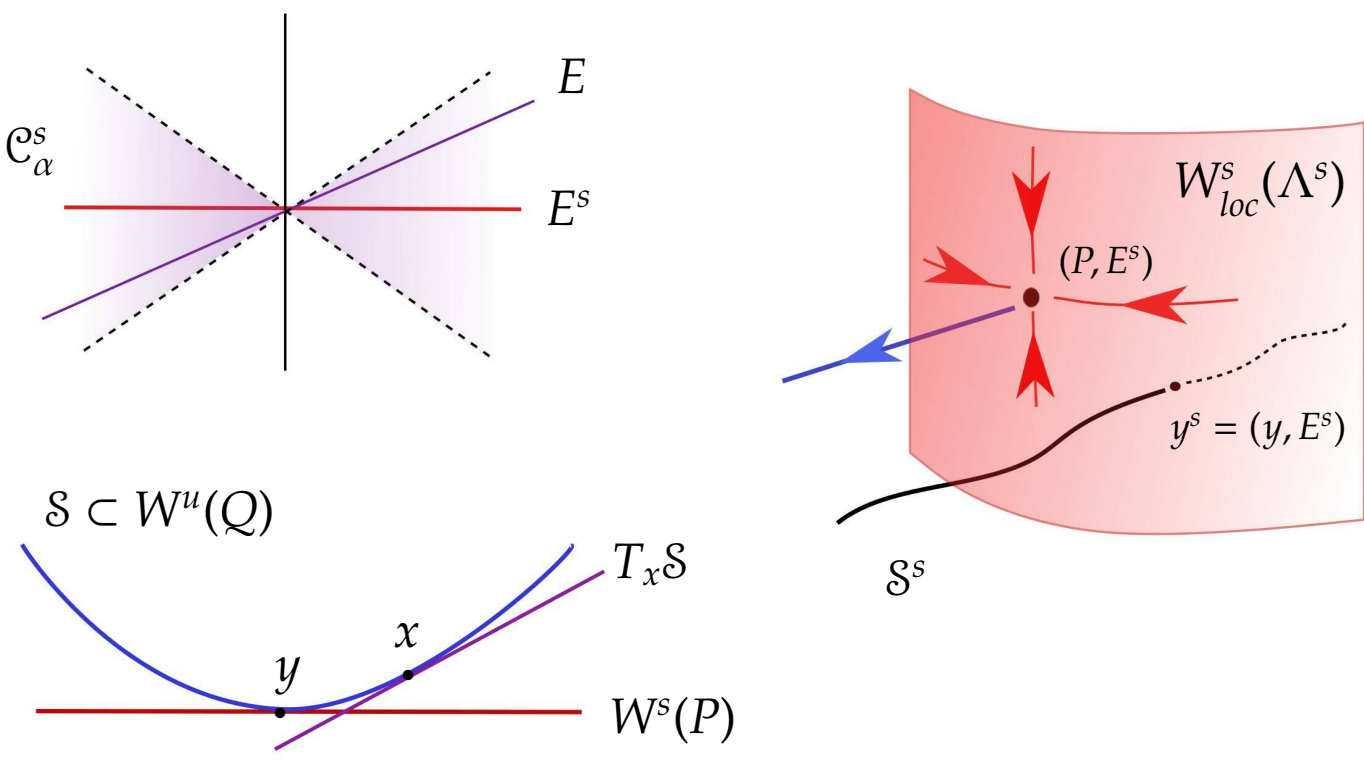

$\mathcal{S}^{s}$

Figure 5. Figure on the left shows the folding manifold $\mathcal{S}$ on $\mathcal{M}$ and how cover the cone $\mathcal{C}_{\alpha}^{s}$ on $\mathbb{R}^{d}$. The other shows the transverse intersection between $\mathcal{S}^{s}$ and the stable manifold of $\Lambda^{s}$ on $\mathbb{R}^{d} \times G(s, d)$

\section{Differential construction of $C^{1}$-robust heteroclinic tangencies}

In this section we will prove again Theorem A but now using a different argument. This different approach allows us to generalize the result to get robust heterodimensional tangencies of large codimension in the next section. In order to explain the idea behind of this new approach we will consider again the situation described in Figure 2.

By considering, if necessary, local coordinates around $P$, define the projective cocycle $f^{s}(x, E)=(f(x), D f(x) E)$ where $x \in \mathbb{R}^{2}$ and $E$ belongs to the space $G(1,2)$ of one-dimensional vector space in $\mathbb{R}^{2}$. Recall that the $\Lambda$ is a hyperbolic attractor of $f$ with splitting $E^{S} \oplus E^{u}$. Hence, the set $\Lambda^{s}=\Lambda \ltimes E^{s}=\left\{(x, E): x \in \Lambda, E=E^{s}(x)\right\}$ is a hyperbolic set of $f^{s}$ where the direction corresponding to the variable in $G(1,2)$ is uniformly expanding. Thus, $W^{s}\left(\Lambda^{s}\right)=$ $W^{s}(\Lambda) \ltimes E^{s}=\left\{(x, E): x \in W^{s}(\Lambda), E=E^{s}(x)\right\}$ is a two-dimensional manifold in the threedimensional space $\mathbb{R}^{2} \times G(1,2)$ as it is showed in Figure 5. On the other hand the unstable manifold of $Q$ contains a folding manifold that we denote by $\mathcal{S}$. That is, a small piece of the unstable manifold contained the point $y$ in its interior. Namely, this manifold folds with respect to the stable cone-field of $f$ at the point $P \in \Lambda$ as it is represented Figure 5 . That is, by considering linear transport to the origin of $\mathbb{R}^{2}$, the union of tangent spaces $T_{x} S$ where $x \in \mathcal{S}$ cover the cone $\mathcal{C}_{\alpha}^{\mathcal{S}}=\{(x, y):|y| \leq \alpha|x|\}$ for some small $\alpha>0$. This property allows us to see the set $\mathcal{S}^{S}=S \ltimes T S=\left\{(x, E): x \in S, E=T_{x} \mathcal{S}\right\}$ as a graph of a function $E \in \mathfrak{C}_{\alpha}^{s} \mapsto x=x(E) \in \mathcal{S}$. In other words, as a one-dimensional manifold in $\mathbb{R}^{2} \times G(1,2)$ which is the image of a graph over $G(1,2)$ and thus transversally intersecting $W^{s}\left(\Lambda^{s}\right)$ at the point 
$\left(y, E^{S}\right)$ where $E^{S}=E^{S}(P)=\mathbb{R} \times\{0\}$. Since this intersection is transversal, it persists for any small perturbation. In particular, for any small perturbation $g$ of $f$, we get a intersection point between $\mathcal{S}^{s}$ and $W^{s}\left(\Lambda_{g}^{s}\right)$ where $\Lambda_{g}^{s}$ is the continuation of $\Lambda^{s}$ for cocycle $g^{s}$ induced by $g$ in $\mathbb{R}^{2} \times G(1,2)$. Notice that this intersection point between $\mathcal{S}^{s}$ and $W^{s}\left(\Lambda_{g}^{s}\right)$ provides the tangency point and direction between $\mathcal{S}$ and a stable manifold $W^{s}(z)$ for some $z \in \Lambda_{g}$. Therefore, we get a robust tangency.

3.1. Construction. Now we will give the formal details. Recall the $C^{r}$-diffeomorphism $f: \mathcal{M} \rightarrow \mathcal{M}$ in (1) and the attractor $\Lambda=\left\{0^{d-2}\right\} \times \Sigma$ in (2). This set has a well defined hyperbolic structure $T_{\Lambda} \mathcal{M}=E^{s} \oplus E^{u}$ where the stable bundle $E^{s}$ of $\Lambda$ is $(d-1)$-dimensional. Observe that $E^{s}$ can be uniquely extended to a continuous $D f$-invariant fiber bundle, which we also denote by $E^{s}$, over each leaf $W_{l o c}^{s}(x), x \in \Lambda$, and so to the whole set $D_{\varepsilon}$. Moreover, from the hyperbolicity of $\Lambda$, we have that $E^{s}$ varies continuously with respect to the point $x \in D_{\varepsilon}$ and the diffeomorphism $g$ in a small $C^{1}$ neighborhood $\mathcal{V}$ of $f$. Thus, for each $g \in \mathcal{V}$ the set $D_{\varepsilon}$ is foliated by $(d-1)$-dimensional (local) stable manifolds of $\Lambda_{g}$ which are tangent to the bundle $E_{g}^{S}$ continuation of $E^{s}$.

Fix $s \stackrel{\text { def }}{=} d-1$. On the set $D_{\varepsilon}$ we have defined a stable cone-field $\mathcal{C}_{\alpha}^{s}$ of dimension $s$ and size $\alpha>0$ satisfying

$$
E^{s}(x) \in \mathcal{C}_{\alpha}^{s}(x) \subset T_{x} \mathbb{R}^{d} \text { and } \quad D f^{-1}(x) \mathcal{C}_{\alpha}^{s}(f(x)) \subset \mathcal{C}_{\alpha}^{s}(x) \quad \text { for all } x \in D_{\varepsilon} .
$$

In what follows, for notational simplicity, we omit the subscript $\alpha$ in the notation $\mathcal{C}_{\alpha}^{s}$. The sdimensional cone-field $e^{s}$ can be seen as an open set of the Grassmannian manifold $G_{s}\left(\mathbb{R}^{d}\right)=$ $\mathbb{R}^{d} \times G(s, d)$ where $G(s, d)$ is set of the s-planes in $\mathbb{R}^{d}$. Observe that in the case $d=2$, this Grassmannian manifold is the projective space. Now consider the differential cocycle induced by $f$ on $G_{s}\left(\mathbb{R}^{d}\right)$ given by

$$
f^{s}: G_{s}\left(\mathbb{R}^{d}\right) \rightarrow G_{s}\left(\mathbb{R}^{d}\right), \quad f^{s}(x, E)=(f(x), D f(x) E) .
$$

Observe that $f^{s}$ is a $C^{r-1}$-diffeomorphism of $G_{s}\left(\mathbb{R}^{d}\right)$ with $r \geq 2$. Since $E^{s}$ is a repelling point of $D f$,

$$
\Lambda^{s}=\Lambda \ltimes E^{S} \stackrel{\text { def }}{=}\left\{(x, E): x \in \Lambda, E=E^{S}(x)\right\}
$$

is hyperbolic set of $f^{s}$ with stable index equals to $\operatorname{dim} E^{s}=s$. Namely, the splitting of $\Lambda^{s}$ is of the form $E^{s} \oplus E^{u} \oplus E^{u u}$ where $E^{s} \oplus E^{u}$ corresponds with the splitting of $\Lambda$ for $f$ and $E^{u u}$ with the directions over $G(s, d)$. On the other hand, the local stable manifold $W_{l o c}^{s}\left(\Lambda^{s}\right)$ of $\Lambda^{s}$ contains the set

$$
D_{\varepsilon}^{s}=D_{\varepsilon} \ltimes E^{S} \stackrel{\text { def }}{=}\left\{(x, E): x \in D_{\varepsilon}, E=E^{S}(x)\right\} .
$$

This is a manifold of codimension the dimension of $G(s, d)$.

We now construct the heteroclinic tangency. First, we give a more formal notion of heterodimensional tangency between any two manifolds.

Definition 3.1. Let $\mathcal{L}$ and $\mathcal{N}$ be two submanifolds of $\mathcal{M}$. We say that $\mathcal{L}$ and $\mathcal{N}$ has a heteroclinic tangency at $x \in \mathcal{L} \cap \mathcal{N}$ if $c_{T}=d_{T}-k_{T}>0$ where

$$
d_{T}=\operatorname{dim} T_{x} \mathcal{L} \cap T_{x} \mathcal{N} \text { and } k_{T}=\operatorname{dim} \mathcal{L}+\operatorname{dim} \mathcal{N}-\operatorname{dim} \mathcal{M} .
$$


The numbers $d_{T}=d_{T}(x, \mathcal{L}, \mathcal{N}), c_{T}=c_{T}(x, \mathcal{L}, \mathcal{N})$ and $k_{T}=k_{T}(x, \mathcal{L}, \mathcal{N})$ are called, respectively, dimension, codimension and signed co-index of the tangency between $\mathcal{L}$ and $\mathcal{N}$ at $x$. The tangency is said to be heterodimensional if $k_{T} \neq 0$ and equidimensional if $k_{T}=0$.

For simplicity and clarity of the exposition we restrict the construction to the case of signed co-index $k_{T}=s-1=d-2$. By means of a similar argument one can also get the other possible co-index in Theorem A. We will consider two types of tangencies: elliptical (see Section 2) and of saddle type. We recall that a tangency $y \in W^{u}(Q) \cap W^{s}(P)$ is of saddle type if every neighborhood $U$ of $y$ contained in either, $W^{u}(Q)$ or $W^{s}(P)$, say $W^{u}(Q)$, intersects each connected component of $\mathbb{R}^{d} \backslash T_{y} W^{u}(Q)$.

Example 3.2. Consider a diffeomorphism having two periodic saddles $P$ and $Q$ such that $0^{d} \in W_{l o c}^{s}(P) \subset \mathbb{R}^{s} \times\{0\}$, with $0^{d} \neq P$ and $\operatorname{dim} W^{s}(Q)=s$. Assume that, $\mathcal{S}\left([-1,1]^{s}\right) \subset W^{u}(Q)$, where

$$
\mathcal{S}:\left(t_{1}, \ldots, t_{s}\right) \mapsto\left(t_{1}, \ldots, t_{s}, t_{1}^{2}+\cdots+t_{s}^{2}\right)
$$

or

$$
\mathcal{S}:\left(t_{1}, \ldots, t_{s}\right) \mapsto\left(t_{1}, \ldots, t_{s}, t_{1} t_{2}+\cdots+t_{s-1} t_{s}\right) .
$$

Then $0^{d}$ is a heteroclinic tangency of elliptic type between $W^{s}(P)$ and $W^{s}(Q)$ choosing $\mathcal{S}$ as in (3) and the saddle type if $\mathcal{S}$ is as in (4).

As it is usual, we identified the embedding $\mathcal{S}$ (as those described above) with its image. Now, using the $s$-dimensional manifold $\mathcal{S}$ in (3) and (4) we create a tangency between the leaves the foliation of $D_{\varepsilon}$ by stable manifold (of dimension $s$ ) of $\Lambda$. Fix a fixed point $P \in \Lambda$ and consider $y \in W_{l o c}^{s}(P)$ with $y \neq P$. Modifying slightly the construction of the attractor if necessary, we can consider coordinates $\left(t_{1}, \ldots, t_{d}\right)$ in neighborhood of $P$ such that

- $P$ is identified with $\left(1^{d-1}, 0\right)$ and $y$ with $0^{d}$,

- the local unstable manifold $W_{l o c}^{u}(P)$ is $t_{1}=\cdots=t_{d-1}=1$,

- for each $z=\left(1^{d-1}, t\right) \in W_{l o c}^{u}(P)$, the local stable manifold $W_{l o c}^{s}(z)$ is $t_{d}=t$; and

- the bundle $E^{S}$ is trivial on this neighborhood.

Hence, in this local coordinates we can assume that $E^{s}=\mathbb{R}^{s} \times\{0\}$ and

$$
\mathcal{C}^{s}=\left\{(u, v) \in \mathbb{R}^{s} \oplus \mathbb{R}:\|v\|<\alpha\|u\|\right\} \cup\left\{0^{d}\right\}
$$

where $\alpha>0$ is a small constant.

At this coordinates, the folding manifolds $\mathcal{S}$ in (3) and (4) intersect $W^{s}(P)$ at $y$ in a heteroclinic tangency of codimension $c_{T}=1$ and signed co-index $k_{T}=s-1=d-2$. The next result state that this tangency persist under perturbations.

Proposition 3.3. The folding manifold $\mathcal{S}$ has a heteroclinic tangency with the stable foliation of $\Lambda$ which persists under small $C^{1}$-perturbations of $f$.

The proof of this proposition makes use of the following result: 
Lemma 3.4. The set $\mathcal{S}^{S}=S \ltimes T S \stackrel{\text { def }}{=}\left\{(x, E): x \in \mathcal{S}, E=T_{x} \mathcal{S}\right\}$ is a manifold of dimension $\operatorname{dim} G(s, d)$ embedded as a disc in $\mathbb{R}^{d} \times G(s, d)$. Namely it is a graph of a function of the form $E \in \mathcal{C}^{\mathcal{S}} \mapsto x=x(E) \in \mathcal{S}$.

Let us postpone for a while the proof of lema, to conclude the proof of the proposition.

Proof of Proposition 3.3. Since $\mathcal{S}$ tangentially meets $W_{l o c}^{s}(P)$ at $y$, we get that $\mathcal{S}^{s}$ topologically transversally intersect $W_{l o c}^{s}\left(\Lambda^{s}\right)$ at $y^{S}=\left(y, E^{S}\right)$. The (topological) transversality follows from Lemma 3.4 since $\mathcal{S}^{s}$ is a disc of $\operatorname{dim} G(s, d)$ and $W_{l o c}^{s}\left(\Lambda^{s}\right)$ is a manifold of codimension $\operatorname{dim} G(s, d)$. See Figure 5 .

Now consider a diffeomorphism $g C^{1}$-close to $f$. Observe that the cocycle $g^{s}$ is a homeomorphism of $G_{s}\left(\mathbb{R}^{d}\right)$ only $C^{0}$-close to $f^{s}$. However, $\Lambda_{g}^{s}=\Lambda_{g} \ltimes E_{g}^{s}$ is still a topological hyperbolic set for $g^{s}$ where $\Lambda_{g}$ and $E_{g}^{s}$ are the continuation of $\Lambda$ and $E^{s}$ for $g$. Thus, the set $W^{s}\left(\Lambda_{g}^{s}\right)$ contains a manifold $C^{0}$-close to $W_{l o c}^{s}\left(\Lambda^{s}\right)$ of codimension $\operatorname{dim} G(s, d)$. Thus we still have a transversal intersection between $\mathcal{S}^{s}$ and $W_{l o c}^{s}\left(\Lambda_{g}^{s}\right)$. Observe that if $(x, E) \in \mathcal{S}^{S} \cap W_{l o c}^{s}\left(\Lambda_{g}^{s}\right)$ then $x \in \mathcal{S} \cap W_{\text {loc }}^{S}\left(\Lambda_{g}\right), E=E_{g}^{S}(x)$ and $E=T_{x} \mathcal{S}$. Thus $E_{g}^{S}(x)=T_{x} \mathcal{S}$. This provides a tangency between $\mathcal{S}$ and the stable foliation of $\Lambda_{g}$ concluding the proof of the proposition.

Remark 3.5. Proposition 3.3 also holds for any small enough $C^{1}$-perturbation of $\mathcal{S}$. To see this, if the perturbation is $C^{1}$-close then we have a change of variable $C^{1}$-close to the identity sending the perturbed manifold to the folding manifold $\mathcal{S}$. Hence we get a new diffeomorphism $g$ which is $C^{1}$-close to $f$. Thus, applying Proposition 3.3 we get a tangency.

Theorem A follows from the above proposition and remark by considering that the folding manifold is contained in the unstable manifold of a hyperbolic fixed point of $f$ of unstable index $s=d-1$. Observe that the codimension of the tangency is given by the formula $c_{T}=d_{T}-k_{T}$ where $d_{T}$ is the number of tangent directions and $k_{T}$ is the co-indice between the hyperbolic set involved. In this case, $c_{T}=s-(s-1)=1$ and $k_{T}=s-1=d-2$.

To complete our construction we give the proof of Lemma 3.4.

Proof of Lemma 3.4. To prove that $\mathcal{S}^{s}$ is an embedded disc in $\mathbb{R}^{d} \times G(s, d)$ we need to show that $\mathcal{S}^{S}$ is a graph of an injective function of the form

$$
\mathcal{S}^{s}: E \in \mathcal{C}^{s} \mapsto x=x(E) \in G(s, d) .
$$

To do this, we must associate to $E$ an unique point $x \in \mathcal{S}$ such that $E=T_{x} \mathcal{S}$. In other words, we need to show that

$$
\mathcal{C}^{\mathcal{S}} \subset \bigcup_{x \in \mathcal{S}} T_{x} \mathcal{S}
$$

As above, we are standing that $\mathcal{C}^{s}$ is a small open set in $G(s, d)$ centered at $E^{s}=\mathbb{R}^{s} \times\{0\}$ and the tangent space $T_{x} \mathcal{S}$ as a vector space of $\mathbb{R}^{d}$. Analytically, we need to solve the following problem: given $E \in \mathcal{C}^{s}$ we look for $t=\left(t_{1}, \ldots, t_{s}\right)$ such that $E=T_{x} \mathcal{S}$ where $x=\mathcal{S}(t)$. 
In order to do the calculation, we choose the elliptic form of the folding manifold given in (3). For folding manifold of saddle type in (4) the argument is similar. Hence,

$$
T_{x} \mathcal{S}:\left(t_{1}^{\prime}, \ldots, t_{s}^{\prime}\right) \in \mathbb{R}^{S} \mapsto\left(t_{1}^{\prime}, \ldots, t_{s}^{\prime}, 2 t_{1} t_{1}^{\prime}+\cdots+2 t_{s} t_{s}^{\prime}\right) \in \mathbb{R}^{d}, \quad \text { where } x=\mathcal{S}\left(t_{1}, \ldots, t_{s}\right) .
$$

We write $E=\operatorname{span}\left\langle v_{1}, \ldots, v_{s}\right\rangle$ where $v_{i}=\left(a_{1 i}, \ldots, a_{d i}\right)$ for $i=1, \ldots, s$. Hence $E=T_{x} \mathcal{S}$ if, and only if, $v_{i} \in T_{x} \mathcal{S}$ for all $i=1, \ldots, s$. Equivalently, if

$$
t_{j i}^{\prime}=a_{j i} \text { for } j=1, \ldots, s \text { and } 2 t_{1} t_{1 i}^{\prime}+\cdots+2 t_{s} t_{s i}^{\prime}=a_{d i} \text { for all } i=1, \ldots, s .
$$

Hence,

$$
2 \cdot\left(\begin{array}{ccc}
a_{11} & \ldots & a_{1 s} \\
& \ddots & \\
a_{s 1} & \ldots & a_{s s}
\end{array}\right) \cdot\left(\begin{array}{c}
t_{1} \\
\vdots \\
t_{s}
\end{array}\right)=\left(\begin{array}{c}
a_{d 1} \\
\vdots \\
a_{d s}
\end{array}\right)
$$

That is, we have a square linear system $A t=b$ where $A=A(E)$ and $b=b(E)$ depends on the vector space $E$. To find $t$ we need to show that $A$ is an invertible matrix. To do this, we will take as the vector space $E$ the center $E^{S}=\mathbb{R}^{S} \times\{0\}=\operatorname{span}\left\langle e_{1}, \ldots e_{S}\right\rangle$ of $\mathcal{C}^{S}$ where $e_{i}$ denotes the vector with a 1 in the $i$-th coordinate and 0 's elsewhere. We get in this case that $A\left(E^{S}\right)=2 \cdot I_{S}$ where $I_{S}$ is the identity square matrix of order $s$. Thus $\operatorname{det} A\left(E^{S}\right) \neq 0$. Then by the continuity for all $E \in \mathcal{C}^{s}$ close to $E^{S}$ we uniquely solve (5) and thus we find $t=\left(t_{1}, \ldots, t_{s}\right)$ such that $E=T_{x} \mathcal{S}$ where $x=\mathcal{S}(t)$. This completes the proof of the lemma.

\section{4. $C^{1}$-ROBUST HETERODIMENSIONAL TANGENCIES OF LARGE CODIMENSION}

Fix $c_{T} \geq 1$ and $s>c_{T}$. Set $d=c_{T} \cdot(s+1)$. A hyperbolic set $\Lambda$ of a diffeomorphism of a manifold $M$ is said to be a codimension one expanding attractor if for every $x \in \Lambda$, holds that $W^{u}(x) \subset \Lambda$ and $\operatorname{dim} W^{u}(x)=\operatorname{dim} M-1$. Let us take a codimension one expanding hyperbolic attractor $\Lambda$ of a diffeomorphism $h$ on a manifold of dimension $n=d-s+1$. In order to avoid the problem of classifying the manifold that support these kind of attractors, we set $\Sigma$ as the Derived from Anosov (by short $D A$-attractor) in the $n$-torus $\mathbb{T}^{n}$, see [15]. After that, we will consider a $C^{r}$ diffeomorphism $f$ of $\mathbb{T}^{d}$ locally defined on $D_{\varepsilon}=[-\varepsilon, \varepsilon]^{s-1} \times \mathbb{T}^{n}$ for a fixed small $\varepsilon>0$ and $r \geq 2$ as

$$
f=g \times h \text { where } g(t)=\lambda t \text { for } t \in[-\varepsilon, \varepsilon]^{s-1} \text { and } 0<\lambda<1 .
$$

Notice that the set $\Lambda=\left\{0^{s-1}\right\} \times \Sigma$ is a hyperbolic attractor of $f$ whose basin of attraction contains $D_{\varepsilon}$. Moreover, $E^{s}=\mathbb{R}^{s-1} \times \tilde{E}^{s}$ is the stable bundle of $\Lambda$ where $\tilde{E}^{s}$ is the onedimensional stable bundle of $\Sigma$ for $h$. Thus, $s=\operatorname{dim} E^{s}$. Analogously as in previous sections, this bundle can be uniquely extended to a $D f$-invariant bundle over $D_{\varepsilon}$ which we also denote by $E^{s}$. Consequently the set $D_{\varepsilon}$ is foliated by s-dimensional stable manifolds of $\Lambda$ which are tangent to $E^{s}$. This allows us to consider a stable cone-field $e^{s}$ of dimension $s$ defined in whole $D_{\varepsilon}$. As in Section 3, we defined the differential cocycle $f^{s}$ induced by $f$ on $G_{s}\left(\mathbb{R}^{d}\right)$. Similarly, we have that the set $\Lambda_{f}^{s}=\Lambda \ltimes E^{s}$ is also a hyperbolic set of $f^{s}$ with stable index equals to $s$ and whose local stable manifold $W_{l o c}^{s}\left(\Lambda_{f}^{s}\right)$ contains the set $D_{\varepsilon}^{s}=D_{\varepsilon} \ltimes E^{s}$. Thus, this manifold has by codimension the dimension of $G(s, d)$. 
Restricting us to a small ball $B \subset D_{\varepsilon}$, we can assume that the stable cone is give by

$$
\mathcal{C}^{s}=\left\{(u, v) \in \mathbb{R}^{s} \oplus \mathbb{R}^{d-s}:\|v\|<\alpha\|u\|\right\}
$$

where $\alpha>0$ is small enough and $E^{s}=\mathbb{R}^{s} \times\left\{0^{d-s}\right\}$. We will consider a folding manifold $\mathcal{S}$ in $B$ folded with respect to $\mathcal{B}$ which we introduce formally as follows:

Definition 4.1. A manifold $\mathcal{S}$ of dimension $k \geq s$ is called folding manifold in an open ball $B$ folded with respect to the cone $\mathrm{C}^{\mathrm{s}}$ if $\mathcal{S} \subset B$ and

$$
\overline{\mathcal{C}^{s}} \subset \bigcup_{x \in \mathcal{S}} T_{x} \mathcal{S}
$$

We are understanding that $\overline{\mathcal{C}^{s}}$ is closure of the open set $\mathcal{C}^{s}$ in $G(s, d)$ centered at $E^{s}$ which we see as a cone in $\mathbb{R}^{d}$ and the tangent space $T_{x} \mathcal{S}$ as a $k$-dimensional vector space of $\mathbb{R}^{d}$. Taking $\alpha$ tends to zero we observe that the above definition is in fact an infinitesimal property of $\mathcal{S}$. Thus without restriction we can assume that the tangent space of $\mathcal{S}$ covers injectively the closure of $\mathcal{C}^{s}$. This means that for every $E \in \mathcal{C}^{s}$ we have a unique $x \in \mathcal{S}$ such that $E \leq T_{x} \mathcal{S}$. Moreover, $x=x(E)$ varies continuously with respect to $E$.

Example 4.2. Take $k=d-c_{T}=c_{T} \cdot s$. Let us consider a $k$-dimensional manifold $\mathcal{S}$ defined by

$$
\mathcal{S}:\left(t_{1}, \ldots, t_{k}\right) \mapsto\left(t_{1}, \ldots, t_{k}, t_{1}^{2}, t_{2}^{2}, \ldots, t_{c_{T}-1}^{2}, t_{c_{T}}^{2}+\cdots+t_{k}^{2}\right) .
$$

Hence, we have that

$$
T_{x} \mathcal{S}:\left(t_{1}^{\prime}, \ldots, t_{s}^{\prime}\right) \mapsto\left(t_{1}^{\prime}, \ldots, t_{k^{\prime}}^{\prime} 2 t_{1} t_{1}^{\prime}, \ldots, 2 t_{c_{T}-1} t_{c_{T}-1}^{\prime}, 2 t_{c_{T}} t_{c_{T}}^{\prime}+\cdots+2 t_{k} t_{k}^{\prime}\right),
$$

where $x=\mathcal{S}\left(t_{1}, \ldots, t_{k}\right)$. We write $E=\operatorname{span}\left\langle v_{1}, \ldots, v_{s}\right\rangle \in \mathcal{C}^{s}$ where $v_{i}=\left(a_{1 i}, \ldots, a_{d i}\right)$ for $i=1, \ldots, s$. Hence $E \leq T_{x} \mathcal{S}$ if, and only if, $v_{i} \in T_{x} \mathcal{S}$ for all $i=1, \ldots, s$. Equivalently, if

$$
\begin{gathered}
t_{j i}^{\prime}=a_{j i} \quad \text { for } j=1, \ldots, k \text { and } 2 t_{\ell} t_{\ell i}^{\prime}=a_{k+\ell i} \text { for } \ell=1, \ldots, c_{T}-1 \text { and } \\
2 t_{c_{T}} t_{c_{T}}^{\prime}+\cdots+2 t_{k} t_{k i}^{\prime}=a_{d i} \quad \text { for all } i=1, \ldots, s .
\end{gathered}
$$

This defines a linear system of $c_{T} \cdot s$ equations and $k$ variable. Since $k=c_{T} \cdot s$ we can write the system in the form $A t=b$ where $A=A(E)$ is a square matrix of ordem $k$ and $b=b(E)$ is a vector in $\mathbb{R}^{k}$ depending on the vector space $E$. To find $t=\left(t_{1}, \ldots, t_{k}\right)$ we need to show that $A$ is an invertible matrix. To do this, we will take as the vector space $E$ the center $E^{s}=\mathbb{R}^{s} \times\{0\}=\operatorname{span}\left\langle e_{1}, \ldots e_{s}\right\rangle$ of $\mathcal{C}^{s}$ where $e_{i}$ denotes the vector with a 1 in the $i$-th coordinate and $0^{\prime}$ s elsewhere. We get in this case that $\operatorname{det} A\left(E^{S}\right)=2$. Then, by continuity, for all $E \in \mathcal{C}^{s}$ close to $E^{S}$ we uniquely solve the equation $A t=b$ and thus we find $t=\left(t_{1}, \ldots, t_{s}\right)$ such that $E \leq T_{x} \mathcal{S}$ where $x=\mathcal{S}(t)$. Therefore $\mathcal{S}$ is folding manifold with respect to $\mathcal{C}^{s}$.

As a consequence of the definition of folding manifold we get the following lemma:

Lemma 4.3. Let $\mathcal{S}$ be a folding manifold folded with respect to $\mathcal{C}^{s}$. Then the set

$$
\mathcal{S}^{s} \stackrel{\text { def }}{=}\left\{(x, E): x \in \mathcal{S}, E \leq T_{x} \mathcal{S} \text { with } \operatorname{dim} E=s\right\}
$$

contains a manifold of dimension $\operatorname{dim} G(s, d)$ embedded as a disc in $\mathbb{R}^{d} \times G(s, d)$. 
Proof. From the definition of folding manifold, we have an injective continuous function $E \in \mathcal{C}^{\mathcal{S}} \mapsto x \in \mathcal{S}$ such that $E \leq T_{x} \mathcal{S}$. This defines a subset of $\mathcal{S}^{\mathcal{S}}$ which is an embedding given by $E \in \mathcal{C}^{s} \mapsto(x, E) \in \mathcal{S} \times G(s, d)$ proving the lemma.

The following result is the analogous to Proposition 3.3.

Proposition 4.4. Let $\mathcal{S}$ be a folding manifold in $B$ of dimension $k=d-c_{T}=c_{T} \cdot s$ folded with respect to $\mathcal{C}^{\mathrm{s}}$. Then $\mathcal{S}$ has a heterodimensional tangency of codimension $\mathcal{c}_{T}$ and signed co-index $k_{T}=s-c_{T}>0$ with the stable foliation of $\Lambda$ which persists under small $C^{1}$-perturbations of $f$.

Proof. By assumption if $x \in B$ then $E^{s}(x)=\mathbb{R}^{s} \times\left\{0^{d-s}\right\} \in \mathcal{C}^{s}$. Thus, we have that $\mathcal{S}$ has a heterodimensional tangency of codimension $c_{T}$ with $W^{S}(z)$ for some $z \in \Lambda$. Indeed, by definition of the folding manifold $\mathcal{S}$ and the stable bundle $E^{\mathcal{S}}$ we find $x \in \mathcal{S} \subset B$ and $z \in \Lambda$ such that $E^{S}(x) \leq T_{x} \mathcal{S}$ and $E^{S}(x)=T_{x} W^{S}(z)$. Furthermore, the signed co-index of the tangency is $k_{T}=s+k-d=k-1=s-c_{T}>0$ and the codimension is $d_{T}-k_{T}=s-\left(s-c_{T}\right)=c_{T}$. On the other hand, the point $\left(x, E^{s}(x)\right)$ belongs to $\mathcal{S}^{c} \cap W^{s}\left(\Lambda^{s}\right)$. Moreover, from Lemma 4.3, we have that $\mathcal{S}^{s}$ contains a disc of dimension $\operatorname{dim} G(s, d)$. Additionally, $W^{s}\left(\Lambda^{s}\right)$ has codimension $\operatorname{dim} G(s, d)$. Hence $\mathcal{S}^{s}$ transversally intersect (in a topological sense) $W^{s}\left(\Lambda^{s}\right)$.

Arguing as in Proposition 3.3, we still have a transversal intersection between $\mathcal{S}^{s}$ and $W^{s}\left(\Lambda_{g}^{s}\right)$ for any $C^{1}$-close diffeomorphism $g$ to $f$. Thus there is $(x, E) \in \mathcal{S}^{s} \cap W^{s}\left(\Lambda_{g}^{s}\right)$. Then $x \in \mathcal{S} \cap W^{s}\left(\Lambda_{g}\right), E \leq T_{x} \mathcal{S}$ and $E=T_{x} W^{s}(z)$ for some $z \in \Lambda_{g}$. Similar as above, this implies that $\mathcal{S}$ and $W^{S}(z)$ has a heterodimensional tangency of codimension $c_{T}$ and signed co-index $k_{T}=s-c_{T}$ concluding the proof of the proposition.

Proof of Theorem B. It suffices to consider that the folding manifold in Proposition 4.4 is contained in the unstable manifold of a hyperbolic fixed point of $f$ of unstable index $k$.

\section{Discussion AND OPEN QUeSTIONS}

The goal of this paper was to construct heteroclinic tangencies which are robust under $C^{1}$ perturbations. This question was proposed in [12, pag. 3281] where the authors showed the existence of $C^{2}$-robust heterodimensional tangencies. To approach this problem we have constructed $C^{1}$-robust tangencies where one of the hyperbolic sets involved is an attractor. This limitation prevents that our construction could be carried on a heterodimensional cycle. A diffeomorphism has a heterodimensional cycle associated with two transitive hyperbolic sets if these sets have different indices (dimension of the stable bundle) and their invariant manifolds meet cyclically. This cycle is called non-transverse (heterodimensional) cycle if besides its cyclic intersections involves some heterodimensional tangency. In order to construct a robust non-transverse heterodimensional cycle one must construct the tangency involving hyperbolic sets which are not attractors. This leads to our first question:

Question 1. Is it possible to construct $C^{1}$-robust non-transverse heterodimensional cycles? 
Bearing in mind the classic constructions of robust homoclinic tangencies and heterodimensional cycles $([19,7])$ via the unfolding of tangencies and cycles associated with saddles, we ask the following:

Question 2. Can a diffeomorphism $f$ having a non-transverse heterodimensional cycle associated with saddles $P$ and $Q$ be $C^{r}$-approximated by a diffeomorphism $g$ with a $C^{r}$-robust non-transverse heterodimensional cycle associated with hyperbolic sets containing the continuations $P_{g}$ and $Q_{g}$ of $P$ and $Q$ ?

On the other hand, we also deal in this paper with the construction of heterodimensional tangencies with signed co-index $k>0$ of large codimension. Robust tangencies of large codimension were discovered in [3]. Namely, the authors provided a method to construct $C^{2}$-robust bundle tangencies which are non-trivial intersection between different fiber bundles. Bundle tangencies include homoclinic, heterodimensional and equidimensional tangencies. Recently in [4], using similar ideas similar to this paper, we have constructed new examples of robust homoclinic tangencies of large codimension. The construction also uses an abstract notion of folding manifold with respect to a cone-field extending previous approach on robust homoclinic tangencies in [5]. However, as in the case of this work, the construction are limited to consider high dimensional manifolds. The lower possible dimension that allows to have a homoclinic tangency of large codimension is $d=4$. Similarly, $d=5$ is the lower dimension to construct a large heterodimensional tangency with signed co-index $k>0$. Thus we address the following questions:

Question 3. Is it possible to build a robust heterodimensional tangency with signed co-index $k>0$ (resp. homoclinic tangency) of codimension $c_{T}=2$ in dimension $d=5$ (resp. $\left.d=4\right)$ ?

Acknowledgements. We are grateful to Artem Raibekas for discussions and helpful suggestions. During the preparation of this article PB was supported by MTM2017-87697-P from Ministerio de Economía y Competividad de España and CNPQ-Brasil. SP were partially supported by CMUP (UID/MAT/00144/2019), which is funded by FCT with national (MCTES) and European structural funds through the programs FEDER, under the partnership agreement PT2020. SP also acknowledges financial support from a postdoctoral grant of the project PTDC/MAT-CAL/3884/2014

\section{ReFERENCES}

[1] Abraham, R. and Smale, S. Nongenericity of $\Omega$-stability. Global Analysis Proc. Sympos. Pure Math., Vol. XIV, Berkeley, Calif., 1968, 5-8.

[2] Asaoka, M. Hyperbolic sets exhibiting $C^{1}$-persistent homoclinic tangency for higher dimensions. Proc. Amer. Math. Soc., 136, (2008), 677-686.

[3] Barrientos, P. G. and Raibekas, A. Robust tangencies of large codimension. Nonlinearity, 30, (2017), 4369-4409.

[4] Barrientos, P. G. and Raibekas, A. Robust nongeneric unfolding of cycles and tangencies. preprint (2017).

[5] Bonatti, C. and Díaz, L. J. Abundance of $C^{1}$-homoclinic tangencies. Trans. Amer. Math. Soc., 264 (2012), 5111-5148.

[6] Bonatti, C. and Díaz, L. J. On maximal transitive sets of generic diffeomorphisms. Publ. Math., Inst. Hautes Étud. Sci., 96 (2003), 171-197. 
[7] Bonatti, C. and Díaz, L. J. Robust heterodimensional cycles and $C^{1}$-generic dynamics. J. Inst. Math. Jussieu, 7 (2008), 469-525.

[8] Díaz, L. J. and Kiriki, S. and Katsutoshi, S. Blenders in centre unstable Hénon-like families: with an application to heterodimensional bifurcations. Nonlinearity, 27 (2014), 353-378.

[9] Díaz, L. J., Nogueira, A. and Pujals, E. R. Abundance of $C^{1}$-homoclinic tangencies. Trans. Amer. Math. Soc., 264 (2012), 5111-5148.

[10] Díaz, L. J. and Pérez, S. A. Hénon-like families and blender-horseshoes at non-transverse heterodimensional cycles. To appear in: Internat. J. Bifur. Chaos Appl. Sci. Engrg.

[11] Hirsch, M. W., Pugh, C. C. and Shub, M. Invariant manifolds. Bull. Amer. Math. Soc., 76 (1970), 1015-1019.

[12] Kiriki, S. and Soma, T. $C^{2}$-robust heterodimensional tangencies. Nonlinearity, 25 (2012), 3277.

[13] Palis, J. A differentiable invariant of topological conjugacies and moduli of stability. Dynamical systems, Vol. III-Warsaw, 335-346. Astérisque, No. 51, Soc. Math. France, Paris, 1978.

[14] Simon, Carl P. A 3-dimensional Abraham-Smale example. Proc. Amer. Math. Soc., 34 (1972), 629-630.

[15] Smale, S. Differentiable dynamical systems. Bull. Amer. Math. Soc., 73 (1967), 747-817.

[16] Robinson, C. Dynamical systems: stability, symbolic dynamics, and chaos. CRC press, 1998.

[17] Mañé, R. A proof of the $C^{1}$ stability conjecture. Inst. Hautes Études Sci. Publ. Math., 66 (1988), 161-210.

[18] Nitecki, Z. On semi-stability for diffeomorphisms. Inventiones mathematicae, 14 (1971), 83-122.

[19] Newhouse, S. E. The abundance of wild hyperbolic sets and nonsmooth stable sets for diffeomorphisms. Inst. Hautes Études Sci. Publ. Math., 50 (1979), 101-151.

[20] Williams, R. F. The "DA" maps of Smale and structural stability. Global Analysis (Proc. Sympos. Pure Math., Vol. XIV, Berkeley, Calif., 1968, 329-334.

Instituto de Matemática e Estatística, UFF

Rua Prof. Marcos Waldemar de Freitas Reis, s/N, Niterói, Brazil

E-mail address: pgbarrientos@id.uff.br

Centro de Matematica da Universidade do Porto

Rua do Campo Alegre 687, 4169-007 Porto, Portugal

E-mail address: sebastian.opazo@fc.up.pt 